\title{
BMJ Open Effectiveness of smart living environments to support older adults to age in place in their community: an umbrella review protocol
}

\author{
Thomas Tannou (D , , ,2,3,4 Thomas Lihoreau, ${ }^{2}$ Mireille Gagnon-Roy, ${ }^{4,5}$ \\ Myrian Grondin, ${ }^{5}$ Nathalie Bier ${ }^{4,5}$
}

To cite: Tannou T, Lihoreau T, Gagnon-Roy M, et al. Effectiveness of smart living environments to support older adults to age in place in their community: an umbrella review protocol. BMJ Open 2022;12:e054235. doi:10.1136/ bmjopen-2021-054235

- Prepublication history for this paper is available online. To view these files, please visit the journal online (http://dx.doi. org/10.1136/bmjopen-2021054235).

Received 06 June 2021 Accepted 10 January 2022

Check for updates

(c) Author(s) (or their employer(s)) 2022. Re-use permitted under CC BY-NC. No commercial re-use. See rights and permissions. Published by BMJ.

${ }^{1}$ Laboratoire de Recherches Intégratives en Neurosciences et Psychologie Cognitive, Université de Bourgogne Franche Comté (UBFC), Besançon, France

${ }^{2}$ Inserm CIC 1431, University Hospital of Besançon (CHU), Besançon, France

${ }^{3}$ Geriatrics department, University Hospital of Besançon (CHU), Besançon, France

${ }^{4}$ Research Center, Institut Universitaire de Gériatrie de Montréal, Montréal, Quebec, Canada

${ }^{5}$ Ecole de réadaptation, Université de Montréal, Montreal, Quebec, Canada

Correspondence to Dr Thomas Tannou; thomas.tannou@umontreal.ca

\section{ABSTRACT}

Introduction Frailty is a vulnerable condition exposing older adults to incidental adverse health events that negatively impact their quality of life and increase health and social costs. Digital solutions may play a key role in addressing this global problem and in particular, smart living environments. Smart living environments involve a notion of measurement or collection of data via several sensors, capturing the person's behaviours in the home or the person's health status over a long period of time. It thus has great potential for home support for older adults. The objective of this umbrella review will be: (1) to document the effectiveness of smart living environments to support ageing in place for frail older adults and (2) among the reviews assessing the effectiveness of smart living environment, to gather evidence on what factors and strategies were identified as influencing the implementation process.

Methods and analysis We will include systematic and scoping reviews of both quantitative and qualitative primary studies with or without meta-analysis focusing on assessing the effectiveness of interventions through smart living environments to support older adults in the community to age in place. The literature search will be done through the following biomedical, technological and sociological citation databases: MEDLINE, Embase, CINAHL, Web of Science and PsycINF0, and quality assessment of the reviews will be done thought AMSTAR2 checklist. The analysis of the results will be presented in narrative form.

Ethics and dissemination Our review will rely exclusively on published data from secondary sources and will thus not involve any interactions with human subjects. The results will be presented at international conferences and publications.

PROSPERO registration number CRD42021249849.

\section{INTRODUCTION}

Frailty is a vulnerable condition exposing older adults to incidental adverse health events that negatively impact their quality of life and increase health and social costs. ${ }^{1}$ Frailty increases cognitive impairment risk, ${ }^{2}$ is directly associated with impairment in daily living activities ${ }^{3}$ and with a risk of dementia, ${ }^{4}$
Strengths and limitations of this study

- Summarising the effectiveness of smart living environments to support ageing in place for frail older people is a challenge requiring an umbrella review approach.

- Overlap of primary publications could have potential impact on data synthesis and will be analysed.

- Recommendation will be presented according to GRADE (Grading of Recommendations Assessment, Development and Evaluation) and GRADEConfidence in Evidence from Reviews of Qualitative research recommendations.

- Some recent work may not be included as our umbrella review is based on systematic and scoping reviews.

questioning the ability to age in place. Frailty is affecting 32 million Europeans ${ }^{5}$ and 1.5 million Canadians. ${ }^{6} 7$ In coming decades, its prevalence will continue to rise due to an increased life expectancy around the world. The COVID-19 pandemic has reinforced the importance of rethinking how we take care of these frail older adults and exploring innovative solutions related to eHealth. ${ }^{8}$ The COVID-19 pandemic has exposed frail older adults to a higher risk of medical complications as well as social exclusion and isolation due to lockdown and social distancing measures. ${ }^{9} 10$ In particular, the disruption of the usual support and care ecosystems at home has paradoxically increased the frailty of these people and led to the occurrence of adverse events we wanted to avoid. ${ }^{11}$ Moreover, this pandemic has overstretched already limited health professional resources and restricted healthcare professionals from seeing older adults, as day programmes as well as outpatient and home services are put on hold and professionals are deployed to what may be considered higher priority 
urgent care roles. ${ }^{12}$ Consequently, the question of optimising and prioritising health resources is crucial and should be at the heart of postpandemic research efforts.

Digital solutions may play a key role in addressing this global problem ${ }^{13}$ and in particular, smart living environments. ${ }^{14}$ Smart living environments refer to smart homes, that is, to 'environments that adopts ICT (information and communication technology) to collect and share information, analyze and monitor residents' behavioral patterns, and improve residents' quality of life' ${ }^{15} \mathrm{~A}$ 'home' may refer to a single-family house, an apartment or other type of living situation that is not integrated into a care structure (such as nursing homes).

More specifically, smart living environments involve a notion of measurement or collection of data via several sensors, capturing the person's behaviours in the home or his/her health status over a long period of time. This function is referred to as 'remote monitoring' or 'telemonitoring'. Telemonitoring can collect data on the different domains of health components (eg, mobility, cognition) and independence, that is, on activities of daily living (ADL) functions such as cooking or going to the bathroom, based on a combination of different markers such as the level of activity in each room, cupboards that are opened and closed, the entrances and exits of the person into each room or in and out of the house, etc. ${ }^{16}$ Smart living environments also refer to a notion of integration and real-time analysis of the data collected, via machine learning and artificial intelligence, and therefore of prediction or detection of specific events. After detection of a potential adverse event (eg, a fall), the smart environment can send information to a caregiver or to the person living in this environment (eg, notifications to the older adult (eg, possible dehydration) or to other entities outside of the person's home (eg, a neighbour or healthcare professional) ) - involving a notion of telecare.

There have been several breakthroughs in remote home monitoring over the years, especially for continuous physiological data monitoring (detection or prevention). For instance, urinary tract infections in older adults have been detected by monitoring the frequency of bathroom visits. ${ }^{17}$ Studies have also shown the possibility of detecting mild cognitive impairments or dementia such as Alzheimer's disease based on simple markers such as walking speed ${ }^{18-20}$ or time spent completing ADL. ${ }^{21}{ }^{22}$ In this respect, a recent literature review reported that cognitive deficits could be detected by smart home monitoring of general activity, outings, sleep habits and computer usage. ${ }^{23}$ Other studies have shown that the information provided by this monitoring can be very useful to guide clinicians working in home care services. Indeed, it can help them build their intervention plan with personalised real-life data, in order to mobilise the right service at the right time. ${ }^{24}$ Studies have also shown high degrees of concordance between the information gathered by the smart environment and real data on a longitudinal basis. ${ }^{25}$

Smart environment thus has great potential for home support for older adults. According to Siegel $e t a l,{ }^{26}$ smart
Box 1 Reseach question

Obj 1: to document the effectiveness of smart living environments to support ageing in place for frail older adults.

Q1. What is the level of evidence that Smart Homes (SH)/ ambient assisted living (AAL) can support older adults at home?

Q2. How was the level of evidence determined?

Q3. How was this evidence measured in terms of type of outcomes?

Obj 2: among the reviews assessing the effectiveness of smart living environment, to gather evidence on what factors and strategies were identified as influencing the implementation process.

Q4. What are the implementation strategies that were used to deploy such system in real-life settings?

Q5. What are the obstacles of implementing SH/AAL in real-life settings? Q6. What types of technologies are included in SH/AAL?

Q7. What is the information that is used from this system and how?

Q8. Who is the main user of SH/AAL data to support older adults at home?

Q9. How is the data used by the main user? (eg, clinical decision, etc). Q10. Who are the other actors involved? (eg, healthcare system, caregivers, neighbours, etc)

homes are, in fact, highly suited to optimise supportive actions by care organisations such as social and healthcare services. In the last 20 years, a growing number of studies have been dedicated to developing such smart living environments with the purpose of supporting ageing in place. As such, literature reviews on the subject have exploded recently, exploring the effectiveness of smart living environment from different perspectives.

In an effort to guide future research and implementation of smart living environments in healthcare systems, a summarisation of the actual evidence is timely. To do so, umbrella reviews are recognised as a rigorous tool to provide comprehensive overviews of evidence published in the literature. ${ }^{27}$ More specifically, it is a review of systematic reviews and meta-analysis. ${ }^{28}$ Therefore, the objective of this umbrella review will be: (1) to document the effectiveness ${ }^{29}$ of smart living environments to support ageing in place for frail older adults and (2) among the reviews assessing the effectiveness of smart living environment, to gather evidence on what factors and strategies were identified as influencing the implementation process. To this end, we organise our research question into 10 items, presented in box 1 .

\section{METHODS AND ANALYSIS \\ Study design}

We will conduct an umbrella review, defined as 'the compilation of evidence from pre-existing systematic reviews into one accessible and usable document'. ${ }^{27}$ Umbrella reviews focus on research question or clinical practice for which there is a rich and high-quality evidence base. ${ }^{30}$ Umbrella reviews are similar to intervention reviews, but the unit of search, inclusion and data analysis is the review rather than the primary study. ${ }^{31}$ Giving the number of publications and reviews in smart home for older adults 


\section{Box 2 Inclusion/exclusion criteria}

\section{Inclusion criteria}

Publication type, date and language

- Reviews published in a peer-reviewed journal or conference proceedings.

- Reviews published before 1 June 2021.

- Reviews published in English or French.

Study design

- For quantitative reviews: scoping or systematic reviews including a reproducible, systematic search strategy, with clear definition of inclusion/exclusion criteria and outcome.

- For qualitative reviews: reviews including a reproducible, systematic search strategy, with clear inclusion/exclusion criteria definition and structured synthesis plan.

Population

- Reviews concerning older adults (age $>65$ ) living at home with telemonitoring.

Intervention

- Reviews focusing on interventions based on Smart Living Environment (behaviour remote monitoring with unobtrusive devices) to support ageing in place, AND assessing their effectiveness to the target population or caregivers.

\section{Outcome}

- Reviews including effectiveness of smart living environment implemented on older adults houses, and among them, if available, strategies impacting the implementation process.

\section{Exclusion criteria}

- Reviews of interventions focusing exclusively on medical monitoring.

- Reviews focusing exclusively on non-health related outcomes, such as ethical issues or acceptability of technology without implementation processing.

- Reviews focusing solely on the use of smart homes by caregivers, if they did not consider determining the efficiency.

these last years, the overview provided by this kind of analysis suits well to examine whether the evidence for our research question is available ${ }^{32}$ and whether it is consistent or contradictory. ${ }^{33}$ Indeed, the rigorous analysis of potential discrepancies in the selection keywords of or inclusion criteria and/or overlapping studies that would lead to analysis bias ${ }^{34}$ allows to assess how authors who have worked independently on the same question have been able to obtain a consistent or inconsistent approach. By listing all the reviews on the subject, we will be able to give a global view of the leading articles on the research question.

This umbrella review will be reported in accordance with box 2 of the Preferred Reporting Items for Overviews of Review statement protocol, ${ }^{35}$ such as the description of the objectives, Population Intervention Comparator Outcome (PICO) criteria for eligibility, methods used for all steps of the screening, selection and extraction, quality assessment, management of overlap, analysis and synthesis, in order to allow replicability of our analysis.

Our study covers review articles published before 1 June 2021 and is expected to end before mid-2022.

\section{Target population}

We will include systematic and scoping reviews of both quantitative and qualitative primary studies with or without meta-analysis focusing on assessing the effectiveness of interventions through smart living environments to support older adults in the community to age in place. The full list of inclusion and exclusion criteria developed for this umbrella review is provided in box 2 .

In our umbrella study, the term 'smart living environment' refers to any unobtrusive monitoring systems used to analyse and share information about residents' behaviours to enable ageing in place. ${ }^{15}$ 'Unobtrusive technology' refers to sensor-based technology that does not draw the user's attention or demand their direct involvement and that blends well into the environment. ${ }^{3637}$ This definition thus excludes wearables, smartphones and camera-based systems. ${ }^{36}$ Given the lack of a consensual term, all similar terminology, such as 'health smart home', 'telemonitoring' or 'ambient assisted living' will potentially be included if the remote monitoring objectives correspond to the population criteria. By 'older adults', we will focus on adults aged 65 and over that have been included in a study using a smart living environment that detects everyday behaviour and share information to the older adult itself and/or caregivers (or other types of stakeholders). As we focus on ageing in place of adults over 65 years, we will not look at smart devices used for medical monitoring with specific diseases such as diabetes or cardiovascular disease (eg, level of blood sugar, blood pressure, temperature).

We will also delimit 'home' as individual houses with or without informal caregivers. To assess the level of evidence for these smart environments, we will specifically include reviews that focus on the implementation of these devices. We will exclude reviews measuring exclusively non-health related outcomes, such as ethical issues or acceptability of technology, as well as reviews that focus solely on the use of these smart homes by caregivers, if they did not consider determining the effectiveness.

\section{Intervention, comparators and outcomes}

Reviews will be included if they provide synthesised results on the effectiveness of a smart living environment to support frail older adults to age in place. Among them, we will focus on evidence of the factors and strategies that have been identified as influencing the implementation process. We will examine results from reviews reporting all types of non-invasive smart living environments (public or private), whether they are single or combined technologies, and whether they are provided alone or within a circle of support including formal and informal caregivers.

In those reviews where a comparator is required, any comparator(s) tested will be considered: smart living environment versus control (no specific environment, usual care, caregivers' intervention, placebo or other control, or another intervention) as defined by the original reviews. We will include different resident and 
caregivers' outcomes (satisfaction, burden risk, impact on cognitive status, etc), since we want to give an overview of the different outcome measures used in the included reviews.

\section{Publication type, date and language}

Reviews published in a peer-reviewed journal or conference will be included, without date limitation. We will restrict the inclusion to reviews published in languages spoken by research team members, namely English and French.

\section{Data sources and search strategy}

An iterative search strategy for bibliometric databases will be developed to look for peer-reviewed systematic reviews evaluating smart living environment to support older adults to age in place, including quantitative and/ or qualitative syntheses of implementation studies and process evaluations alongside trials testing such technology. The search for this umbrella review will aim to identify all research syntheses relevant to our research questions. The literature search will be done through the following biomedical, technological and sociological citation databases: MEDLINE, Embase, CINAHL, Web of Science and PsycINFO. The search strategy will be guided by a librarian, comprehensively reported and the detailed search filters employed will be presented sequentially in the single appendix for all the databases that were searched and listed along with the search dates.

The preliminary search strategy is detailed in box 3 . This search strategy was developed by the research team with advice from experienced health science and new technology librarians to estimate the number of potentially relevant systematic reviews. Additional searches will be developed for syntheses of effectiveness, implementation or process evaluations published or commissioned by official health agencies. We also expect that a significant number of these reports meeting our criteria will not necessarily be published in peer-reviewed journals or conference proceedings. Finally, we will manually search the reference lists of relevant journals.

\section{Data collection}

In line with recommendations to ensure the objectivity of systematic reviews, titles and citation abstracts will be reviewed by two independent, blinded reviewers using Covidence software (https://www.covidence.org/) developed by the Cochrane collaboration. Any discrepancies will be analysed by a third reviewer. Prior to selection, the inclusion criteria were tested in a pilot study. Similarly, the eligibility of the full texts of relevant papers will be independently checked by two independent reviewers and any disagreements will be resolved following reading by a third reviewer. Reviews excluded during the full-text review will be documented with the reason for exclusion.

\section{Methodological quality and risk of bias assessment}

Critical appraisal of quantitative reviews will be conducted independently by two reviewers using the AMSTAR-2

\section{Box 3 Search strategy}

1. exp "review"/.

2. exp "Review Literature as Topic"/.

3. exp meta-analysis/.

4. exp meta-analysis as topic/.

5. exp "Systematic Review"/.

6. exp Systematic Reviews as Topic/.

7. ("review* of reviews" or "overview or reviews" or "meta-analy" or metaanaly* or "meta-review*" or metareview* or "metha-synthe*" or metasynthe* or "meta-ethnograph" or metaethnograph* or "research evidence" or "scoping of the evidence*" or "scoping project*" or "mapping exercise" or "systematic map" or "mixed method" review" or "mixed method* overview" or "mixed method" summar" "or "mixed stud" review" "or "mixed stud" overview" or "mixed stud" summar" or "mixed research review" or "mixed research overview" or "mixed research summar" or "expedited review*" or "expedited overview*" or "expedited summar*").ab,kf,ti.

8. ((systematic or state-of-the-art or scoping or literature or umbrella or rapid or mapping or integrative or synthesis or evidence*) adj3 (review* ${ }^{*}$ or overview* ${ }^{*}$ or assessment* or summar ${ }^{*}$ or study or studies or report* or synthesis)).ab,kf,ti.

9. 1 or 2 or 3 or 4 or 5 or 6 or 7 or 8 .

10. ("home automat*" or domotic* or "embedded health* system*" or "ambient* assisted" or "ambient* living" or "ambient* intelligen*" or "artificial intelligence*" or "assistive technolog*" or "digital ecosystem*" or "software ecosystem*" or "tech* ecosystem*" or gerontechnolog* or telesurveillance or "tele surveillance" or telemonitoring or "tele monitoring").ab,kf,ti.

11. ((smart or intelligen* ${ }^{*}$ or adapted or adaptive or adaptative or aware or ambient* or embedded or integrated) adj2 (home* or house* or housing or environment* or residen*)).ab,kf,ti.

12. ((smart or intelligen* or aware or ambient* or embedded or integrated) adj2 system*).ab,kf,ti.

13. 10 or 11 or 12 .

14. exp Aged/.

15. exp Health Services for the Aged/.

16. exp Aging/.

17. exp Housing for the Elderly/.

18. exp Geriatrics/.

19. exp Independent Living/.

20. exp Personal Autonomy/.

21. exp Caregivers/.

22. $\exp$ Activities of Daily Living/.

23. (senior* or aged or elder* or "old" adult*" or "old* people*" or "old* person*" or frail* or geront* or geriatr" or aging or ageing or autonomy or independenc* or "independent living" or "liv* independen*" or "living alone" or "single household" or "single house hold" or "solo-living" or "stay* at home" or "stay* in place*" or "communitydwelling" or "home-dwelling" or "assisted hous*" or "assisted living" or "support* hous*" or "support* living" or caregiver* or "care giver" or carer* or "self-management*" or "activit* of daily living" or "every day activit"” or "everyday activit"”).ab,kf,ti.

24. 14 or 15 or 16 or 17 or 18 or 19 or 20 or 21 or 22 or 23 .

25. 9 and 13 and 24.

26. limit 25 to (english or french).

checklist. ${ }^{38}$ The checklist allows the quality of the reviews to be ranked according to seven critical and nine noncritical areas. Based on the assessment, the reviews will 
be grouped into critically low, low, moderate and highquality categories. Any disagreements in the quality assessment process will be resolved through moderated discussions by a third reviewer.

\section{Data extraction}

Data will be extracted independently by the reviewers according to an extraction grid previously tested on five random articles to check the correct completion of the data. To check inter-judge reproducibility, the first five journals will be double extracted, blinded. A discussion between the reviewers will allow the adjustment of the data extraction for the whole corpus.

Extraction will be limited to the data reported in the included reviews, without judging the interpretation from the source studies. Nevertheless, the references included in source studies will be extracted to indicate overlap and avoid bias due to double counting.

\section{Data synthesis}

The analysis of the results will be presented in narrative form and will refer to a detailed table of the characteristics of the included reviews. This presentation will contextualise the results in terms of relevance to supporting ageing in place, technological maturity, elements of implementation and evidence from the research syntheses included in the study, following our research questions (box 1). It will also describe the typologies of technologies used, as well as the different forms of interaction with users (residents, natural caregivers, professional caregivers). The data presented will be based on the elements and criteria used in the included reviews. Data from qualitative research will also be commented and synthesised directly from the primary authors' analyses using thematic trees. In addition, the place of both older adults and caregivers will be analysed to show implication in codesign, if available.

To perform these analyses, the data from the included review will be directly $\operatorname{coded}^{39}$ to obtain summary tables containing, for each of the questions in box 1, reproducible keywords describing the major results of the included reviews. These tables will be included in our result section.

Overlap is a key issue in the reporting of an umbrella review. It can lead to false assumptions about the evidence presented by a disproportion between sources. ${ }^{40}$ It is therefore necessary to present an overlap analysis. In our study, this will be done by a quantitative approach, that is, with the calculation of the corrected covered area. ${ }^{41}$ Additionally, our umbrella review will present, in graphic form, the interaction between the source studies.

Then, the levels of evidence relating to each of the research questions (box 1) will be synthesised using the methodology proposed by the GRADE (Grading of Recommendations Assessment, Development and Evaluation) Working Group for quantitative data ${ }^{42}$ and the GRADE-Confidence in Evidence from Reviews of Qualitative research for qualitative data. ${ }^{43}$

\section{Patient and public involvement}

No patients or members of the public were involved in the development of this umbrella review. However, the scope and methods of this review were based on the literature and discussed with experts from an international scientific consortium of which the authors are members.

\section{Ethics and dissemination}

The proposed umbrella review will rely exclusively on published data from secondary sources and will thus not involve any interactions with human subjects. It is therefore exempt from institutional review board (IRB) approval.

The results from the umbrella review will be presented at international conferences within the fields of, for example, gerontology and geriatrics, health informatics, primary care, public health and social sciences, and will be published in a journal addressing a broad readership. On publication of the results, we will make the data generated by our research openly and publicly available. The team also intends to use social networks and institutional websites from their research centres to publicise its findings via websites, social media, and newsletters.

\section{DISCUSSION}

Ageing in place is the wish of the majority of persons when asked about their future. However, frailty, social isolation, cognitive impairment and the resulting vulnerabilities make ageing in place in the community a complex process. The use of smart living environments to support the analysis of individual behaviours, identify the need for targeted interventions by carers and thus maintain quality of life and support ageing in place seems essential. The COVID-19 pandemic has highlighted the importance of being able to rely on innovative technologies to target the interventions of professionals as well as caregivers, and to support frail older people.

By detailing, through a global approach, all the data available in the literature, our umbrella review will allow research teams as well as policy makers to identify the technologies and implementation strategies that have been successful in supporting ageing at home for older people.

The production of umbrella reviews is becoming more and more common. The aim is to synthesise levels of evidence, for example, to develop clinical guidelines or to support public health or research funding policy orientations. Nevertheless, umbrella reviews present major methodological challenges, both in terms of managing overlap and in terms of classifying recommendations. Through our original approach to the issue of home support for frail older people, we also wish to contribute to these issues.

\section{Twitter Thomas Tannou @TannouThomas}

Contributors TT drafted the protocol. All authors (TT, TL, MG, MG-R and NB) participated in conceptualising the methodology, provided critical revisions and approved the final version of the protocol for submission.

Funding This work was supported by the French National Research Agency (ANR)—-through MRSEl process—grant number: ANR-196MRS3-0025-01.

Competing interests None declared.

Patient and public involvement Patients and/or the public were not involved in the design, or conduct, or reporting, or dissemination plans of this research. 
Patient consent for publication Not applicable.

Provenance and peer review Not commissioned; externally peer reviewed.

Open access This is an open access article distributed in accordance with the Creative Commons Attribution Non Commercial (CC BY-NC 4.0) license, which permits others to distribute, remix, adapt, build upon this work non-commercially, and license their derivative works on different terms, provided the original work is properly cited, appropriate credit is given, any changes made indicated, and the use is non-commercial. See: http://creativecommons.org/licenses/by-nc/4.0/.

\section{ORCID iD}

Thomas Tannou http://orcid.org/0000-0003-3476-9822

\section{REFERENCES}

1 Andrew MK, Dupuis-Blanchard S, Maxwell C, et al. Social and societal implications of frailty, including impact on Canadian healthcare systems. J Frailty Aging 2018;7:1-7.

2 Feng L, Nyunt MSZ, Gao Q, et al. Physical frailty, cognitive impairment, and the risk of neurocognitive disorder in the Singapore longitudinal ageing studies. J Gerontol A Biol Sci Med Sci 2017;72:369-75

3 Kojima G. Quick and simple frail scale predicts incident activities of daily living (ADL) and instrumental ADL (IADL) disabilities: a systematic review and meta-analysis. J Am Med Dir Assoc 2018;19:1063-8.

4 Borges MK, Canevelli M, Cesari M, et al. Frailty as a predictor of cognitive disorders: a systematic review and meta-analysis. Front Med 2019;6.

5 Manfredi G, Midão L, Paúl C, et al. Prevalence of frailty status among the European elderly population: findings from the survey of health, aging and retirement in Europe. Geriatr Gerontol Int 2019;19:723-9.

6 Collard RM, Boter H, Schoevers RA, et al. Prevalence of frailty in community-dwelling older persons: a systematic review. J Am Geriatr Soc 2012;60:1487-92.

7 Pérez-Zepeda MU, Godin J, Armstrong JJ, et al. Frailty among middle-aged and older Canadians: population norms for the frailty index using the Canadian longitudinal study on aging. Age Ageing 2021;50:447-56.

8 Smith AC, Thomas E, Snoswell CL, et al. Telehealth for global emergencies: implications for coronavirus disease 2019 (COVID-19) J Telemed Telecare 2020;26:309-13.

9 Talbot CV, Briggs P. 'Getting back to normality seems as big of a step as going into lockdown': the impact of the COVID-19 pandemic on people with early to middle stage dementia. Age Ageing 2021:50:657-63.

10 Aroos R, Wong BLL, Merchant RA. Delayed health consequences of COVID-19 lockdown in an older adult. Age Ageing 2021;50:673-5.

11 Hollander JE, Carr BG. Virtually perfect? Telemedicine for Covid-19. N Engl J Med 2020;382:1679-81.

12 Koeberle S, Tannou T, Bouiller K, et al. COVID-19 outbreak organisation of a geriatric assessment and coordination unit. A French example. Age Ageing 2020;49:516-22.

13 Carnemolla P. Ageing in place and the internet of things - how smart home technologies, the built environment and caregiving intersect. Visualization in Engineering 2018;6.

14 Li F, Valero M, Shahriar H, et al. Wi-COVID: a COVID-19 symptom detection and patient monitoring framework using WiFi. Smart Health 2021;19:100147.

15 Lee LN, Kim MJ. A critical review of smart residential environments for older adults with a focus on pleasurable experience. Front Psychol 2020;10.

16 Debes C, Merentitis A, Sukhanov S, et al. Monitoring activities of daily living in smart homes: understanding human behavior. IEEE Signal Process Mag 2016;33:81-94.

17 Rantz MJ, Skubic M, Koopman RJ. Using sensor networks to detect urinary tract infections in older adults. e-Health Networking Applications and Services (Healthcom), 2011 13th IEEE International Conference, 2011:1429.

18 Kaye J, Mattek N, Dodge H, et al. One walk a year to 1000 within a year: continuous in-home unobtrusive gait assessment of older adults. Gait Posture 2012;35:197-202.

19 Kaye JA, Maxwell SA, Mattek N, et al. Intelligent systems for assessing aging changes: home-based, unobtrusive, and continuous assessment of aging. J Gerontol B Psychol Sci Soc Sci 2011;66(Suppl 1):i180-90.
20 Akl A, Taati B, Mihailidis A. Autonomous unobtrusive detection of mild cognitive impairment in older adults. IEEE Trans Biomed Eng 2015;62:1383-94.

21 Dawadi PN, Cook DJ, Schmitter-Edgecombe M, et al. Automated assessment of cognitive health using smart home technologies. Technol Health Care 2013;21:323-43.

22 Lussier M, Adam S, Chikhaoui B, et al. Smart home technology: a new approach for performance measurements of activities of daily living and prediction of mild cognitive impairment in older adults. $J$ Alzheimers Dis 2019;68:85-96.

23 Lussier M, Lavoie M, Giroux S, et al. Early detection of mild cognitive impairment with in-home monitoring sensor technologies using functional measures: a systematic review. IEEE J Biomed Health Inform 2019;23:838-847.

24 Fazio S, Pace D, Flinner J, et al. The fundamentals of PersonCentered care for individuals with dementia. Gerontologist 2018;58:S10-19.

25 Rodgers MM, Pai VM, Conroy RS. Recent advances in wearable sensors for health monitoring. IEEE Sens J 2015;15:3119-26.

26 Siegel C, Hochgatterer A, Dorner TE. Contributions of ambient assisted living for health and quality of life in the elderly and care services - a qualitative analysis from the experts' perspective of care service professionals. BMC Geriatr 2014;14.

27 Grant MJ, Booth A. A typology of reviews: an analysis of 14 review types and associated methodologies. Health Info Libr J 2009;26:91-108.

28 Aromataris E, Fernandez R, Godfrey CM, et al. Summarizing systematic reviews: methodological development, conduct and reporting of an umbrella review approach. Int J Evid Based Healthc 2015;13:132-40.

29 Singal AG, Higgins PDR, Waljee AK. A primer on effectiveness and efficacy trials. Clin Trans/ Gastroenterol 2014;5:e45.

30 Aromataris E, Fernandez R, Godfrey C. Chapter 10: Umbrella reviews. In: JBI manual for evidence synthesis. Adelaide, Australia: JBI Collaboration, 2020.

31 Pollock M, Fernandes R, Becker L. Chapter V: Overviews of reviews. In: Cochrane Handbook for systematic reviews of interventions version 62. 2021. Cochrane, 2021

32 Lunny C, Brennan SE, Reid J, et al. Overviews of reviews incompletely report methods for handling overlapping, discordant, and problematic data. J Clin Epidemiol 2020;118:69-85.

33 JSM K, Pollock M, Kaunelis D, et al. Guidance on review type selection for health technology assessments: key factors and considerations for deciding when to conduct a de novo systematic review, an update of a systematic review, or an overview of systematic reviews 2021.

34 Faulkner G, Fagan MJ, Lee J. Umbrella reviews (systematic review of reviews). Int Rev Sport Exerc Psychol 2021;7:1-18.

35 Pollock M, Fernandes RM, Pieper D, et al. Preferred reporting items for Overviews of reviews (PRIOR): a protocol for development of a reporting guideline for overviews of reviews of healthcare interventions. Syst Rev 2019;8:335.

36 Sharma N, Brinke JK, Gemert-Pijnen JEWCV, et al. Implementation of Unobtrusive sensing systems for older adult care: Scoping review. JMIR Aging 2021;4:e27862.

37 Weiser M. Some computer science issues in ubiquitous computing Commun ACM 1993;36:75-84.

38 Shea BJ, Reeves BC, Wells G, et al. AMSTAR 2: a critical appraisal tool for systematic reviews that include randomised or nonrandomised studies of healthcare interventions, or both. BMJ 2017;358:j4008.

39 Hennessy EA, Johnson BT, Keenan C. Best practice guidelines and essential methodological steps to conduct rigorous and systematic Meta-Reviews. Appl Psychol Health Well Being 2019;11:353-81.

40 Lunny C, Pieper D, Thabet P, et al. Managing overlap of primary study results across systematic reviews: practical considerations for authors of overviews of reviews. BMC Med Res Methodol $2021 ; 21: 140$.

41 Pieper D, Antoine S-L, Mathes T, et al. Systematic review finds overlapping reviews were not mentioned in every other overview. $J$ Clin Epidemiol 2014;67:368-75.

42 Guyatt GH, Oxman AD, Vist GE, et al. GRADE: an emerging consensus on rating quality of evidence and strength of recommendations. BMJ 2008;336:924-6.

43 Noyes J, Booth A, Lewin S, et al. Applying GRADE-CERQual to qualitative evidence synthesis findings-paper 6: how to assess relevance of the data. Implement Sci 2018;13:4. 